\title{
Drawing Planar 3-Trees with Given Face-Areas ${ }^{\star}$
}

\author{
Therese Biedl and Lesvia Elena Ruiz Velázquez \\ David R. Cheriton School of Computer Science, University of Waterloo, \\ Waterloo, ON N2L 3G1, Canada \\ \{biedl, leruizve\}@uwaterloo.ca
}

\begin{abstract}
We study straight-line drawings of planar graphs such that each interior face has a prescribed area. It was known that such drawings exist for all planar graphs with maximum degree 3 . We show here that such drawings exist for all planar partial 3-trees, i.e., subgraphs of a triangulated planar graph obtained by repeatedly inserting a vertex in one triangle and connecting it to all vertices of the triangle. Moreover, vertices have rational coordinates if the face-areas are rational, and we can bound the resolution. We also give some negative results for other graph classes.
\end{abstract}

\section{Introduction}

A planar graph is a graph that can be drawn without crossing. Fáry, Stein and Wagner [39:12 proved independently that every planar graph has a drawing such that all edges are drawn as straight-line segments. Sometimes additional constraints are imposed on the drawings. The most famous one is to have integer coordinates while keeping the area small; it was shown in 1990 that this is always possible in $O\left(n^{2}\right)$ area 48. Another restriction might be to ask whether all edge lengths are integral; this exists if the graph is 3-regular [5, but is open in general.

In this paper, we consider drawings with prescribed face areas. This has applications in cartograms, where faces (i.e., countries in a map) should be proportional to some property of the country, such as population. Ringel [7] showed that such drawings do not exist for all planar graphs. Thomassen [10] showed that they do exist for planar graphs with maximum degree 3 . Quite a few results are known for drawings with prescribed face areas that are not straight-line, but instead use orthogonal paths, preferably with few bends [11/1/2].

We show that every planar partial 3-tree, for any given set of face areas, admits a planar straight-line drawing that respects the face areas. It is quite easy to show that such drawings exist; our main contribution is that the coordinates are rational (presuming the face-areas are). This has not been studied before when drawing planar graphs with prescribed face-areas. Furthermore, we can bound the resolution in terms of the number of vertices (albeit not polynomially).

It remains open whether Thomassen's proof could be modified to yield rational coordinates for all planar graphs of maximum degree 3 ; we provide some evidence why this seems unlikely. We also show that some planar partial 4 -tree cannot

\footnotetext{
^ Research supported by NSERC.
} 
be realized at all, and another planar partial 4-tree can be realized only with irrational coordinates.

\section{Background}

Let $G=(V, E)$ be a graph with $n$ vertices and $m$ edges that is simple (has no loops or multiple edges) and planar (can be drawn without crossing.) A planar drawing of $G$ splits the plane into connected pieces; the unbounded piece is called the outer-face, all other pieces are called interior faces. We assume that one combinatorial drawing (characterized by the clockwise order of edges around each vertex and choice of the outer-face) has been fixed for $G$.

A planar straight-line drawing of $G$ is an assignment of vertices to distinct points in the plane such that no two (induced) straight-line segments of edges cross, and the fixed order of edges and outer-face are respected.

Let $A$ be a function that assigns non-negative rational 11 to interior faces of $G$. We say that a planar straight-line drawing of $G$ respects the given face areas if every interior face $f$ of $G$ is drawn with area const $\cdot A(f)$, where the constant is the same for all faces. If $A \equiv 1$, then the drawing is called an equifacial drawing.

A graph $G$ is a $k$-tree if it has a vertex order $v_{1}, \ldots, v_{n}$ such that for $i>k$ vertex $v_{i}$ has exactly $k$ predecessors, i.e., earlier neighbours, and they form a clique. A partial $k$-tree is a subgraph of a $k$-tree. Partial $k$-trees are the same as graphs of treewidth at most $k$; such graphs have received huge attention in the last few years due to the ability to solve many NP-hard problems in polynomial time on graphs of constant treewidth.

Assume $G$ is a planar 3-tree. Then vertex $v_{i}$ (for $i>3$ ) has three predecessors and they form a triangle. Hence we can think of $G$ as being built up by starting with a triangle, and repeatedly picking a face $f$ (which is necessarily a triangle) and subdividing $f$ into three triangles by inserting a new vertex in it. One can show that the first triangle in this process can be presumed to be the outer-face.

A planar partial 3-tree is a graph $G^{\prime}$ that is planar and is the subgraph of a 3-tree $G$ [6]. Planar partial 3-trees include outerplanar graphs, series-parallel graphs, Halin graphs and IO-graphs.

\section{Drawing Planar Partial 3-Trees}

We now show that every planar partial 3-tree can be drawn with given face areas. A vital ingredient is how to draw $K_{4}$ by placing one point inside a triangle.

Lemma 1. Let $T$ be a triangle with area a and vertices $v_{0}, v_{1}, v_{2}$ in counterclockwise order. For any non-negative value $a_{0}+a_{1}+a_{2}=$ $a$, there exists a point $v^{*}$ inside $T$ such that triangle $\left\{v_{i+1}, v_{i-1}, v^{*}\right\}$ has area $a_{i}$, for $i=$ 0,1, 2 and addition modulo 3.

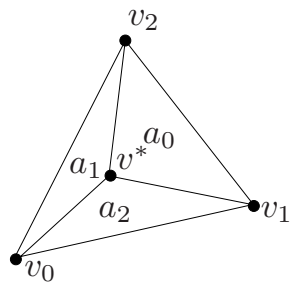

\footnotetext{
${ }^{1}$ Irrational face areas could be allowed, but would force irrational coordinates.
} 
Proof. Let $\left(x_{0}, y_{0}\right),\left(x_{1}, y_{1}\right),\left(x_{2}, y_{2}\right),\left(x^{*}, y^{*}\right)$ be the coordinates of $v_{0}, v_{1}, v_{2}$, $v^{*}$, respectively. The signed area formula expresses the area of a triangle via determinants; the result is positive if the vertices are counterclockwise around the triangle and negative otherwise. In particular, for $a_{i}$ to be the area of a triangle $\left\{v_{i+1}, v_{i-1}, v^{*}\right\}$ (for $i=0,1,2$ and addition modulo 3 ), we must have

$$
\begin{aligned}
2 \cdot a_{i} & =\left|\begin{array}{ccc}
x_{i+1} & y_{i+1} & 1 \\
x_{i-1} & y_{i-1} & 1 \\
x^{*} & y^{*} & 1
\end{array}\right| \\
& =\left(x_{i-1} \cdot y^{*}-x^{*} \cdot y_{i-1}\right)-\left(x_{i-1} \cdot y_{i+1}-x_{i+1} \cdot y_{i-1}\right)+\left(x^{*} \cdot y_{i+1}-x_{i+1} \cdot y^{*}\right)
\end{aligned}
$$

Since the triangle defined by $v_{0}, v_{1}, v_{2}$ has area $a=a_{1}+a_{2}+a_{3}$, we also know

$$
2 \cdot a=\left|\begin{array}{lll}
x_{0} & y_{0} & 1 \\
x_{1} & y_{1} & 1 \\
x_{2} & y_{2} & 1
\end{array}\right|=\left(x_{1} \cdot y_{2}-x_{2} \cdot y_{1}\right)-\left(x_{1} \cdot y_{0}-x_{0} \cdot y_{1}\right)+\left(x_{2} \cdot y_{0}-x_{0} \cdot y_{2}\right)
$$

Combining these equations yields, after sufficient manipulation, that

$$
x^{*}=\frac{a_{1} \cdot x_{1}+a_{2} \cdot x_{2}+a_{3} \cdot x_{3}}{a_{1}+a_{2}+a_{3}} \quad \text { and } \quad y^{*}=\frac{a_{1} \cdot y_{1}+a_{2} \cdot y_{2}+a_{3} \cdot y_{3}}{a_{1}+a_{2}+a_{3}}
$$

Since $2 a_{i}$ is non-negative, the signed-area formula guarantees that $v^{*}$ lies to the left of the directed segments $v_{0} v_{1}, v_{1} v_{2}$, and $v_{2} v_{0}$, and hence inside $T$.

Lemma 2. Every planar 3-tree can be drawn respecting prescribed face areas.

Proof. Assume $v_{1}, \ldots, v_{n}$ is the vertex-order that defined the 3 -tree $G$, with $\left\{v_{1}, v_{2}, v_{3}\right\}$ the outer-face. We proceed by induction on $n$. The base case is $n=3$, where this is obvious. If $n \geq 4$, then consider the $K_{4}$ formed by $v_{n}$ and its neighbours. In $G-v_{n}$, these neighbours form a triangle $T$ that is an interior face. Draw $G-v_{n}$ recursively, requiring as area for $T$ the sum of the area of the faces around $v_{n}$. Then, by Lemma 1, $v_{n}$ can be added inside $T$ suitably.

Lemma 3. Every planar partial 3-tree can be drawn respecting prescribed face areas.

Proof. Recall that a planar partial 3-tree can be augmented into a planar 3-tree $G$ by adding edges. Each time an edge is added, it divides a face $f_{i}$ into two faces $f_{i}^{1}$ and $f_{i}^{2}$. Let $a_{i}$ be the prescribed area for $f_{i}$, then we choose area $a_{i}^{j}$ for face $f_{i}^{j}$ such that $a_{i}^{1}+a_{i}^{2}=a_{i}$, e.g. $a_{i}^{1}=a_{i}^{2}=\frac{a_{i}}{2}$. By Lemma 2 $G$ can be drawn respecting the prescribed face areas. Deleting all added edges then gives the desired drawing.

In our construction, we are interested not only in whether such a drawing exists, but what bounds can be imposed on the resulting coordinates. If all areas are rationals, then Equation (1) shows immediately that the newly placed vertex $v^{*}$ has rational coordinates if the coordinates of $T$ are rational. Hence, using induction and starting in the base case with a triangle with rational coordinates, one can immediately show that all coordinates of all vertices are rational. We summarize: 
Theorem 1. Let $G$ be a planar partial 3-tree and $A$ be an assignment of nonnegative rationals to interior faces of $G$. Then $G$ has a straight-line drawing such that each interior face $f$ of $G$ has area $A(f)$ and all coordinates are rationals.

We can also give bounds on the required resolution.

Theorem 2. Any planar 3-tree $G$ has an equifacial straight-line drawing with integer coordinates and width and height at most $\prod_{k=1}^{n}(2 k+1)$.

Proof. We show that $G$ has an equifacial straight-line drawing with rational coordinates in $[0,1]$ with common denominator at most $\prod_{k=1}^{n}(2 k+1)$; the result then follows after scaling. Let $v_{1}, \ldots, v_{n}$ be a vertex order of $G$ with $v_{1}, v_{2}, v_{3}$ the outer-face. The drawing is the one from Theorem 1 we assume that $v_{1}, v_{2}, v_{3}$ are at the triangle $T=\{(1,0),(0,1),(0,0)\}$ (this can be enforced in the base case of Lemma 2]) Since $G$ is triangulated, it has $2 n-5$ faces; so each interior face is drawn with area $a=1 /(4 n-10)$ since $T$ has area $1 / 2$. We show the bound on the denominator only for $x$-coordinates; $y$-coordinates are proved similarly.

We need some notations. Recall that we can view graph $G$ as being obtained by inserting vertex $v_{j}$ into the triangle $T_{j}$ spanned by the three predecessors of $v_{j}$. Let $G_{j}$ be the subgraph of $G$ induced by all vertices on or inside $T_{j}$. Since $T_{j}$ was a face in the graph induced by $\left\{v_{1}, \ldots, v_{j-1}\right\}$, all vertices in $G_{j}$ are either $v_{j}$, or one of its three predecessor, or a vertex in $\left\{v_{j+1}, \ldots, v_{n}\right\}$ and so $G_{j}$ has at most $n-j+4$ vertices. Let $f_{j}$ be the number of interior faces in $G_{j}$; we have $f_{j} \leq 2(n-j+4)-5=2 n-2 j+3$. Also note that $T_{j}$ contains exactly these $f_{j}$ faces and they all have area $1 /(4 n-10)$, so $T_{j}$ has area $f_{j} /(4 n-10)$.

We will show by induction on $i$ that vertex $v_{i}$ has $x$-coordinate

$$
x_{i}=\frac{\text { integer }}{\prod_{4 \leq j \leq i} f_{j}}
$$

for some integer that we will not analyze further to keep notation simple. Nothing is to show for $i=1,2,3$, since $x_{i}$ is an integer by choice of the points for the outer-face triangle. For $i \geq 4$, let $v_{i_{0}}, v_{i_{1}}, v_{i_{2}}$ be the three predecessors of $v_{i}$.

For $k=0,1,2$, Equation (2) holds for $x_{i_{k}}$ by $i_{k} \leq i-1$ and induction, and expanding with integers $f_{i_{k}+1}, \ldots, f_{i-1}$ yields

$$
x_{i_{k}}=\frac{\text { integer }}{\prod_{4 \leq j \leq i_{k}} f_{j}}=\frac{\text { integer }}{\prod_{4 \leq j \leq i-1} f_{j}}
$$

Equation (11) states that $x_{i}=\left(a_{0} x_{i_{0}}+a_{1} x_{i_{1}}+a_{2} x_{i_{2}}\right) /\left(a_{0}+a_{1}+a_{2}\right)$, where $a_{0}, a_{1}, a_{2}$ are the areas of faces incident to $v_{i}$. For $k=0,1,2$, each $a_{k}$ is the sum of faces in some subgraph, and therefore an integer multiple of $1 /(4 n-10)$. Furthermore, $a_{0}+a_{1}+a_{2}$ is exactly the area of triangle $T_{i}$ spanned by $v_{i_{1}}, v_{i_{2}}, v_{i_{3}}$, which we argued earlier is $f_{i} /(4 n-10)$. Hence, as desired,

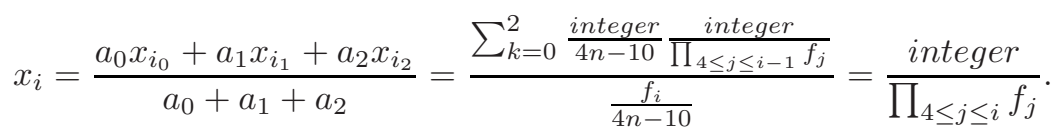


Since $f_{4}, \ldots, f_{n}$ are integers, by Equation (2) all $x_{i}$ 's have common denominator

$$
\prod_{4 \leq j \leq n} f_{j} \leq \prod_{4 \leq j \leq n}(2 n-2 j+3)=\prod_{k=1}^{n-3}(2 k+1)
$$

We mention without proof that we can obtain similar (but uglier-looking) bounds for arbitrary integer face areas, by replacing ' $f_{j}$ ' by 'the sum of the $f_{j}$ largest face areas in $G^{\prime}$ '. We also did experiments to see whether our bounds are tight. We computed (using Maple) the coordinates in Theorem 2 for the planar 3-tree $v_{1}, \ldots, v_{n}$ where $v_{i}$ has predecessors $v_{i-1}, v_{i-2}, v_{i-3}$ for $i \geq 4$; note that this graph has $f_{i}=2 n-2 i+3$ and hence is a good candidate to obtain the bound in Theorem 2. Figure 1 shows the least common denominator for various values of $n$; they are smaller than the upper bound but are clearly growing in exponential fashion as well.

\begin{tabular}{|c|c|c|}
\hline$n$ & LCD in drawing & upper bound \\
\hline \hline 10 & $5.0 \cdot 10^{3}$ & $2.0 \cdot 10^{6}$ \\
\hline 50 & $3.1 \cdot 10^{34}$ & $2.8 \cdot 10^{75}$ \\
\hline 100 & $1.0 \cdot 10^{82}$ & $1.7 \cdot 10^{183}$ \\
\hline 500 & $1.0 \cdot 10^{427}$ & $2.0 \cdot 10^{1271}$ \\
\hline 1000 & $2.8 \cdot 10^{852}$ & $4.8 \cdot 10^{2853}$ \\
\hline
\end{tabular}

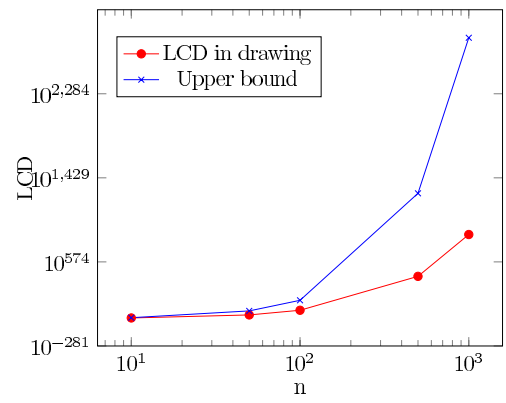

Fig. 1. Lower and upper bounds on the resolution in the drawing

\section{Negative Results}

In this section, we give some examples of graphs where no realization with rational coordinates is possible, hence providing counter-example to some possible conjectured generalizations of Theorem 1 .

The first example is the octahedron where all face areas are 1 except for two non-adjacent, non-opposite faces, which have area 3. As shown by Ringel [7], any drawing that respects these areas must have some complex coordinates. (Ringel's result was actually for the graph $G_{1}$ obtained from the octahedron by subdividing two triangles further; the resulting graph then has no equifacial drawing.) Note that both the octahedron and $G_{1}$ are planar partial 4 -trees, so not all partial 4-trees have equifacial drawings.

The second example is the octahedron where all face areas are 1 except that the three faces adjacent to the outer-face have area 3. (Alternatively, one could ask for an equifacial drawing of graph $G_{2}$ in Figure 2.) Assume, after possible linear transformation, that the vertices in the outer-face are at $(0,0),(0,13)$ and $(2,0)$. Computing the signed area of all the faces one can show that the vertices 

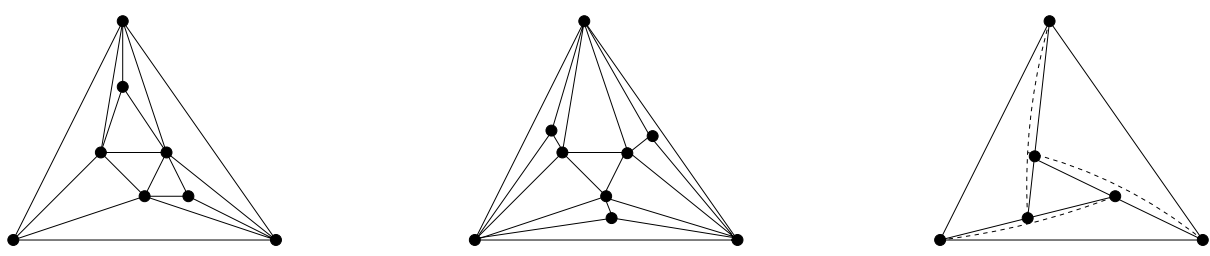

Fig. 2. Graphs $G_{1}, G_{2}$ and $G_{3}$

not on the outerface are at $\left(\frac{10}{3}+\frac{2 \sqrt{3}}{13}, 5-\sqrt{3}\right),\left(\frac{10}{3}-\frac{2 \sqrt{3}}{13}, 3\right)$ and $\left(\frac{6}{13}, 5+\sqrt{3}\right)$. Thus even if a partial 4-tree has an equifacial drawing, it may not have one with rational coordinates.

The third example is again the octahedron, with three face areas prescribed to be 0 , which forces some edges to be aligned as shown in Figure 2. If all other interior faces have area $1 / 8$, and the outer-face is at $(1,0),(0,1),(0,0)$, then similar computations show that some of the coordinates of the other three vertices are $(3 \pm \sqrt{5}) / 8$. Let $G_{3}$ be the graph obtained from the octahedron by deleting the edges that are dashed in Figure 2 Graph $G_{3}$ is a crucial ingredient in Thomassen's proof [10] that every planar of maximum degree 3 graph has a straight-line drawing with given face areas: in one case he splits the input graph into $G_{3}$ and three subgraphs inside three interior faces of $G_{3}$, draws $G_{3}$ with the edges aligned as in Figure 2, and recursively draws and pastes the subgraphs. Since we showed that $G_{3}$ cannot always be drawn with rational coordinates, then Thomassen's proof, as is, does not give rational coordinates. It remains an open problem whether Thomassen's proof could be modified to show that any planar graph with maximum degree 3 has a drawing respecting given rational face-areas that has rational coordinates.

\section{References}

1. de Berg, M., Mumford, E., Speckmann, B.: On rectilinear duals for vertex-weighted plane graphs. In: Healy, P., Nikolov, N.S. (eds.) GD 2005. LNCS, vol. 3843, pp. 61-72. Springer, Heidelberg (2006)

2. Eppstein, D., Mumford, E., Speckmann, B., Verbeek, K.: Area-universal rectangular layouts. In: Proceedings of the 25th Annual Symposium on Computational Geometry. SCG 2009, Aarhus, Denmark, June 08-10, pp. 267-276. ACM, New York (2009)

3. Fáry, I.: On straight line representation of planar graphs. Acta. Sci. Math. Szeged 11, 229-233 (1948)

4. de Fraysseix, H., Pach, J., Pollack, R.: How to draw a planar graph on a grid. Combinatorica 10, 41-51 (1990)

5. Geelen, J., Guo, A., McKinnon, D.: Straight line embeddings of cubic planar graphs with integer edge lengths. Journal of Graph Theory 58(3), 270-274 (2008)

6. Kratochvil, J., Thomas, R.: Manuscript (in preparation)

7. Ringel, G.: Equiareal graphs. In: Contemporary methods in graph theory, in honour of Prof. Dr. K. Wagner, pp. 503-505 (1990) 
8. Schnyder, W.: Embedding planar graphs on the grid. In: 1st Annual ACM-SIAM Symposium on Discrete Algorithms, pp. 138-148 (1990)

9. Stein, S.: Convex maps. Amer. Math. Soc. 2, 464-466 (1951)

10. Thomassen, C.: Plane cubic graphs with prescribed face areas. Combinatorics, Probability \& Computing 1, 371-381 (1992)

11. van Kreveld, M., Speckmann, B.: On rectangular cartograms. In: Albers, S., Radzik, T. (eds.) ESA 2004. LNCS, vol. 3221, pp. 724-735. Springer, Heidelberg (2004)

12. Wagner, K.: Bemerkungen zum Vierfarbenproblem. Jahresbericht der Deutschen Mathematiker-Vereinigung 46, 26-32 (1936) 